\title{
Les anciennes carrières de la région lorientaise
} (Morbihan)

The old quarries of the Lorient area (Morbihan)

\section{Louis Chauris}

\section{(2) OpenEdition}

1 Journals

\section{Édition électronique}

URL : http://journals.openedition.org/abpo/2667

DOI : $10.4000 / a b p o .2667$

ISBN : 978-2-7535-3304-2

ISSN : $2108-6443$

Éditeur

Presses universitaires de Rennes

Édition imprimée

Date de publication : 30 décembre 2013

Pagination : 11-36

ISBN : 978-2-7535-3302-8

ISSN : 0399-0826

\section{Référence électronique}

Louis Chauris, "Les anciennes carrières de la région lorientaise (Morbihan) », Annales de Bretagne et des Pays de l'Ouest [En ligne], 120-4 | 2013, mis en ligne le 30 septembre 2015, consulté le 02 mai 2019. URL : http://journals.openedition.org/abpo/2667 ; DOI : 10.4000/abpo.2667 


\title{
Les anciennes carrières de la région lorientaise (Morbihan)
}

\author{
Louis CHAURIS \\ Directeur de recherche au CNRS (e. r.)
}

Suite aux décisions de Colbert en 1664, Lorient va se développer rapidement $\mathrm{au} \mathrm{XVIII}^{\mathrm{e}}$ siècle sous l'impulsion de la Compagnie des Indes, puis ultérieurement sous l'impact de la Marine, induisant la mise en œuvre d'infrastructures portuaires et défensives, nécessitant de faire appel à de nombreuses carrières. Or, les auteurs qui ont présenté l'histoire de la ville ${ }^{1}$ sont, en règle générale, restés fort discrets sur la nature et la provenance des pierres mises en œuvre, se bornant à des annotations peu précises ${ }^{2}$. Il a semblé qu'un examen lithologique apporterait d'utiles compléments à la connaissance d'une ville appelée à jouer un grand rôle dans l'histoire maritime de la France.

Quelques brefs rappels chronologiques avant d'aborder le thème. L'histoire lorientaise commence en 1666 par l'achat d'une lande au Faouédic sur la rive droite du Scorff en bordure d'une rade (constituée par le confluent de trois rivières : en sus du Scorff, le Blavet et le Ter) servant de refuge aux navires marchands. Lorient, comme Brest, va endurer

1. Parmi d'autres, Chaumell, Louis, "Abrégé de l'histoire de Lorient, de la fondation (1666) à nos jours (1939)" Annales de Bretagne, XlVI, 1939, p. 66-87. Buffet, H. F., " Naissance de la ville de Lorient et son développement sous l'Ancien régime ", Bull. Soc. polym. Morbihan, 1967, p. 21-43. NiĖREs, Claude (dir.), Histoire de Lorient, édit. Privat, Toulouse, 1988, 320 p. (avec une bibliographie commentée). Ibid, Lorient, Morbihan. Atlas historique des Villes de France, édit. CNRS, Paris, 1988. BELSER, Christophe, Lorient. Il y a cent ans en cartes postales anciennes, Édit. Patrimoines-medias, 2006, 156 p.

2. Par exemple : pour la construction de l'église Saint-Louis terminée en 1720 " La Compagnie (des Indes) fournit les pierres de la sacristie ". Après 1732, " ouverture de nombreuses carrières sur les rives des rivières ". "Les premières carrières utilisées se trouvaient sur le Scorff ". De 1735 à 1745, " on pava 8480 toises ", mais la nature des pavés n'est pas indiquée. Parfois, un toponyme émerge : "Plus tard, la Compagnie (des Indes) acheta la perrière de Kergroise ", toutefois le nom de la roche n'est pas précisé. "La Perrière tire son nom d'une grande carrière réputée pour la bonne qualité de ses pierres" dont la composition reste inconnue. 
" la cohabitation du commerce et de la guerre ${ }^{3}$ ». Le premier grand édifice public - l'église Saint-Louis - est achevé en 1720. En 1732, Gabriel fournit ses plans à la Compagnie des Indes; l'hôtel des Ventes est commencé peu après. Dans son état actuel, la tour de La Découverte remonte à 1785. Les fortifications de la ville s'élèvent vers le milieu du XVIII ${ }^{\mathrm{e}}$ siècle, avec deux portes - dites de Hennebont et de Ploemeur - créant une cité " intra-muros ". Le bassin à flot est exécuté entre 1842 et 1847. La qualité des constructions retient l'attention par le souci exigeant de la qualité des pierres utilisées ${ }^{4}$.

Trois remarques encore dans ces propos liminaires. Premièrement, il est évident que les carrières que nous allons évoquer n'ont pas toutes été ouvertes pour les constructions de Lorient. Les communes avoisinantes, beaucoup plus anciennes, avaient longtemps auparavant, fait appel aux roches locales. Que l'on songe aux remparts d'Hennebont, aux belles églises de Larmor-Plage et de Merlevenez... Toutefois, sans la création de Lorient, ces carrières n'auraient eu, ni le nombre, ni l'importance qu'elles allaient acquérir.

Par ailleurs, la région lorientaise n'est pas aussi bien pourvue en pierres que la région brestoise ${ }^{5}$. Ce fait avait été constaté depuis longtemps, comme en témoigne une lettre en date du 15 avril $1778^{6}$ relative aux réparations à faire aux fontaines de Lorient, où il est noté que " la pierre est plus rare à Lorient ", d'où le coût plus élevé des travaux de maçonnerie. Cependant, comme nous allons le montrer, les entreprises ont su, très tôt, se procurer les pierres nécessaires.

Enfin, les documents disponibles sur les anciennes carrières ouvertes dans la région lorientaise sont assez peu nombreux. Les archives du Service historique de la Marine à Lorient conservent un manuscrit de $1741^{7}$ sur les carrières à exploiter pour les constructions à Lorient. Un répertoire (établi en 1836) des carrières du Morbihan, classées par communes regroupées par arrondissement, livre des informations succinctes, parfois assez précises, mais souvent très incomplètes ${ }^{8}$. Le commentaire de la carte géologique du Morbihan (1848) par Th. Lorieux et E. de Fourcy, reflète la compétence

3. NiÈres, Claude, op. cit., note 1.

4. À ce sujet, se reporter, entre autres, à Gérard LE BOUEDEC : «Jusque vers 1850, les ingénieurs des travaux hydrauliques ont imposé leurs conceptions. Les préoccupations architecturales, l'attachement à la symétrie et à l'harmonie transparaissent dans tous les projets de construction. Ce souci d'esthétisme est renforcé par l'environnement architectural hérité de la Compagnie des Indes. On accuse les ingénieurs des travaux hydrauliques de sacrifier [...] les fonds de la Marine à un luxe d'architecture déplacé [...]. Dans les années 1820-1840, les ingénieurs conçoivent des constructions destinées à une durée que le directeur Martret qualifie de perpétuelle. Dans ces conditions, le choix des matériaux alourdit la facture de la construction. La pierre de taille est le matériau roi des édifices ", dans L'information historique, 1994, n 3, p. 94-100.

5. CHAURIS, Louis, "Brest : vieilles pierres et pierres neuves dans une cité reconstruite", Mém. Soc. Hist. et Archéol. de Bretagne, xc, 2012, p. 5-36.

6. Arch. Serv. historique de la Marine à Brest. 1 E 543.

7. Arch. Serv. historique de la Marine à Lorient $1 \mathrm{P} 300$.

8. Arch. dép. du Morbihan S 501. 
des auteurs, ingénieurs au Corps royal des Mines ${ }^{9}$. La carte géologique au 1/80000, feuille "Lorient ", par Ch. Barrois (1885), localise avec précision plusieurs carrières placées dans leur environnement lithologique ${ }^{10}$. Des informations glanées dans archives et publications seront citées plus loin à leur place. Au total, devant le caractère lacunaire de la documentation, est bien vite apparue la nécessité d'enquêtes sur le terrain, facilitées par l'examen stéréoscopique des photographies aériennes.

\section{Un document exceptionnel (1741) sur les carrières à exploiter pour les constructions à l'Orient}

Les nombreuses constructions en cours d'exécution à l'Orient un peu avant la fin de la première moitié du XVIII ${ }^{\mathrm{e}}$ siècle avaient entraîné l'exploitation anarchique de multiples perrières sur les bords du Scorff et du Blavet, dont les décombres déversées sur l'estran risquaient de nuire à la navigation dans la rade. Un extrait des registres du conseil d'État, daté du 31 octobre $1741^{11}$, indique que " l'un des ingénieurs de Sa Majesté, le Sieur Saint-Pierre [s'est] transporté le 8 août dernier et jours suivants" en vue d'examiner " toutes les carrières d'où se tire la pierre dans la rade, ances [sic] du Port-louis, sur les bords des rivières de Blavet, de Scorff ". Sur les 33 carrières reconnues dans ces sites, le Sieur Saint-Pierre " estime qu'il y en a 15 à supprimer tant à cause de leur situation, du préjudice qu'elles pourraient faire par leurs décombres, que par la mauvaise qualité de la pierre qu'elles produisent ". Il propose la conservation de 18 carrières " comme seules utiles tant pour les édifices que la Compagnie des Indes fait construire dans le port, que pour ceux qui se font journellement dans la ville». Les exploitations autorisées se feront en prenant les précautions nécessaires. Il sera statué incessamment " afin que les constructions puissent être continuées sans aucune interruption ". Le Roi " étant en son conseil " permet alors, conformément à l'avis du Sieur Saint-Pierre, l'usage et l'exploitation de 18 carrières dont est présentée la liste, accompagnée dans chaque cas de quelques commentaires.

De ces premières informations quelques points peuvent être déjà soulignés. Trois paramètres ont été pris en compte par l'ingénieur dans le choix des carrières : situation géographique, impact environnemental, qualité de la pierre. On pourra regretter que le nom des carrières délaissées n'ait pas été indiqué; il aurait complété heureusement la documentation sur les extractions aux environs de Lorient vers le milieu du XviII ${ }^{\mathrm{e}}$ siècle. Par ailleurs, on reste assez surpris, à première vue, par le nombre impressionnant de carrières ouvertes dans un terroir somme toute relativement restreint; ce nombre peut s'interpréter par les faibles dimensions de chaque

9. LORIEUX Théodore, FouRcy Eugène de, Carte géologique du Morbihan, Paris, Impr. nat., $1848,157 \mathrm{p}$.

10. BARROIs, Charles, Carte géologique au 1/80000, feuille "Lorient ", 1885.

11. Arch. Serv. historique de la Marine à Lorient 1 P 300. 
carrière, mais aussi par les énormes besoins de la ville en gestation. Quoi qu'il en soit, la liste des carrières retenues s'avère du plus grand intérêt tant par les précisions apportées sur leur localisation que sur leur statut juridique.

1- À la pointe de Kernel, au-delà du ruisseau du moulin du Faouédic. Propriété de M. M. de Faouédic et de Keroman.

2-Sur la lande de Kergrois (aujourd'hui Kergroise), le long des vases de l'anse de Kernel. Abandonnée, naguère exploitée à titre de ferme par un nommé Chaton, "sous les pères de l'Oratoire de Nantes".

3- Au nord de ladite lande, à présent délaissée, autrefois exploitée par ledit Chaton au même titre que la carrière 2 .

4- " Grande carrière à plusieurs ouvertures le long de la côte (au droit) de l'isle de Saint-Michel, dans la lande de Kergrois ".

5- Sur la même côte, jouxtant la précédente, exploitée par un nommé Le Moign.

6- Non loin de la carrière 5, "sur la gauche du passage de Sainte-Catherine ", à présent délaissée, " ci-devant ouverte par le Sieur Le Moign ".

7- À la pointe du Port-Louis, dans la même lande de Kergrois, au midi de l'anse de Keroman, ouverte par le Sieur Chaton et maintenant délaissée. Comme la carrière 2 , les sites des carrières 3 à 7 appartiennent aux pères de l'Oratoire de Nantes, qui les louent à titre de ferme à quelques exploitants. Toutes sont ouvertes sur la rive droite de l'actuelle rade de Pen-Mané, c'està-dire à proximité immédiate de L'Orient.

Les carrières suivantes (8 à 10) situées sur le versant gauche de l'actuelle rade de Port-Louis sont déjà un peu plus éloignées de L'Orient.

8- À la pointe de Kerzo, sur la hauteur, non loin du couvent des Récollets de Sainte-Catherine. La carrière a été ouverte récemment par le Sieur Martinel, entrepreneur à L'Orient.

9- En bas du moulin à vent de "L'Omiquely " (Locmiquelic) près du couvent des Récollets de Sainte-Catherine, ouverte par le Sieur Mangat à titre de ferme sous le Sieur Leyrey.

10- Dans l'anse de "l'Omiquely ", sur la même côte que la précédente carrière, abandonnée à la suite de la mort de l'exploitant, sous le Sieur Leyrey.

Un troisième ensemble de carrières (11 à 14) est encore plus distant.

11- La première est située sur le bord du Blavet, à environ une lieue de L'Orient dans une lande "vis-à-vis le moulin de L'Ocunolé " (Locunolay). Ouverte par le Sieur Guegan "qui dit l'avoir affermé au Sieur de Boblié ".

12- La localisation de la seconde, dénommée Juguerri, située " dans une grande lande ", n'a pu encore être précisée. Abandonnée, elle appartient au Sieur de Gouvelle. Il semblerait qu'elle était ouverte entre "L'Ocunolé " et l'anse de Locoyarn.

13- La troisième, appartenant aussi au Sieur de Gouvillo, est située à proximité de l'anse de Locoyarn.

14- Enfin, la carrière, jouxtant la précédente, appartient à la Compagnie des Indes et est exploitée par l'entrepreneur Charrier. 
Un quatrième et dernier groupe de carrières est situé sur les bords du Scorff.

15- La carrière est ouverte dans une lande au nord du village de Sacqueven (Sac'h Queven) le long de la vasière de K/vegan (Kervegant); elle est exploitée par un nommé Cado à titre de ferme sous le Sieur K/lo-Mauduit.

16- La carrière est située à un quart de lieue au nord de la précédente; elle est affermée par le Sieur Pelletier, de Pont-Scorff. Selon toute probabilité, elle se trouvait près du lieu-dit "Le Roze " où plusieurs carrières abandonnées sont encore visibles aujourd'hui, sur la rive droite du Scorff.

Les deux dernières carrières sont également sur les versants de la rive droite du Scorff, mais nettement plus en aval que les précédentes :

17- Est sur la hauteur, au sud de l'étang du moulin de Faouédic, dans les limites mêmes de la ville de L'Orient; elle appartient à la communauté de ladite ville.

18- Enfin est ouverte au nord du passage de Saint-Christophe, " le long des vases de l'ance [sic] de Querantrey (Kerantrech), vis-à-vis le château de Trisaven (Tréfaren) "; le terrain appartient à la veuve Renou; la carrière a été ouverte par la communauté de L'Orient " pour la chaussée du grand chemin " et " pour les murs à pierres sèches sur les vases du même côté ".

La liste des carrières autorisées est suivie de l'ordonnance du Roi stipulant que « dans trois mois après la publication du présent arrêt, tous les propriétaires, fermiers, entrepreneurs qui voudraient continuer l'exploitation desdites carrières, seront tenus de faire, à leurs frais, tous les quais, parapets et autres ouvrages " pour éviter de porter préjudice aux côtes de la rade en évitant que les décombres sortant des carrières puissent tomber "sur les bords que la mer couvre ", faute de quoi, il sera interdit de continuer l'exploitation des carrières incriminées.

Il était inévitable que des contentieux surgissent à l'occasion des extractions de pierres entre les propriétaires des terrains et les exploitants. À titre d'exemple, voici quelques détails relatifs à la requête présentée par les pères de l'Oratoire de Nantes ${ }^{12}$ qui, comme signalé plus haut, possédaient plusieurs carrières. Le 24 février 1842, la Compagnie des Indes avait acquis du supérieur de la maison de l'Oratoire à Nantes, "l'usage de 300 cordes de carrières " situées au lieu-dit Beg er Groer (?) près de L'Orient. Il était stipulé que la Compagnie des Indes pourrait en jouir " tant que l'on y trouverait de la pierre ". Le terrain fut mesuré, borné et le prix (douze cent quinze livres) fut payé comptant. Or, selon l'Oratoire de Nantes, " depuis ce temps, les carrières dont il s'agit ont été exploitées sans interruption pour le compte de la Compagnie des Indes. Mais au lieu de se renfermer dans les limites convenues par l'acte de 1842, les préposés à l'exploitation ont abattu les bornes posées au midi et dans une des parties du couchant et les ont outrepassées dans l'étendue de plus de 100 cordes ". Depuis 36 ans, les terres ont doublé de valeur, surtout aux environs de Lorient, en conséquence, selon le supérieur de l'Oratoire, il y aurait lieu de " porter à environ

12. Arch. Serv. historique de la Marine à Lorient 1 P 300. 
800 livres l'extraction des 100 cordes de terrain ouvert au-delà des bornes tant pour le prix des pierres qui en ont été tirées que pour le tort résultant de la privation des landes, en grain, pâture ". Mais l'ingénieur ordinaire du port certifiait le 24 septembre 1778 que « l'empiètement actuel au-delà des bornes a été occasionné par les besoins des travaux du Roy postérieurement à la rétrocession faite par la Compagnie des Indes".

\section{Autres données archivistiques du xviiie siècle}

En sus des documents présentés ci-dessus avec quelques détails, les archives du Service historique de la Marine à Lorient renferment également d'autres informations sur les anciennes carrières ouvertes à proximité de Lorient pour les travaux entrepris au XVIII ${ }^{\mathrm{e}}$ siècle. Le devis d'ouvrages à exécuter au port et dans la rade de Lorient, dressé le 5 mai 1770 s'avère particulièrement intéressant :

- les excavations de rochers nécessitées pour les constructions pouvant révéler la présence de bons moellons, il est demandé de les mettre de côté pour être éventuellement employés dans les ouvrages selon les jugements des ingénieurs, louable souci d'économie;

- la carrière de Kergrois (Kergroise) - " dont le Roy a la jouissance " - fait l'objet d'une attention particulière. Cette carrière fournira à l'entrepreneur " les moellons nécessaires pour les ouvrages qui lui seront ordonnés "; ledit entrepreneur mettra à part la pierre qui " ne sera bonne que pour le lestage des vaisseaux ". Par ailleurs, comme l'exploitation de cette carrière fournit " beaucoup d'attraits, de décombres et de pierrailles ", ces éléments seront réservés " pour être employés à la réparation journalière de toutes les digues ». Enfin, l'entrepreneur mettra également de côté « la pierre propre à faire du pavé d'échantillon ". Lesdits pavés seront de la plus grande dimension possible, à savoir longueur de tête depuis 9 pouces jusqu'à 4 pouces au moins, longueur de queue depuis 11 pouces jusqu'à 7 pouces au moins;

- toujours selon le devis du 5 mai 1770, les pierres de taille sont classées en deux catégories. "Les pierres de taille dures " - en l'occurrence, il s'agit de granites - seront tirées des carrières de la côte du Talud, en face de l'île de Groix, distante de deux lieues et demi par terre et par mer; il s'agit en fait du leucogranite de Ploemeur. Les pierres de taille tirées des carrières situées en bordure de la rivière d'Hennebont pourront être aussi utilisées; il n'est toutefois pas précisé ici s'il est question des granites de Polvern en amont d'Hennebont ou de Locoyarn en aval. Quoi qu'il en soit, ces pierres dures ont toutes une origine proximale. Il en est tout autrement des " pierres de taille tendres " - en l'occurrence des calcaires - appelés aussi " pierres blanches ", en provenance du Val de Loire (Saumur), de la Charente (Taillebourg) ou de Normandie (environs de Caen); elles seront choisies " suivant la nature des ouvrages qui pourront être ordonnés ".

Plusieurs autres informations sur les carrières et les pierres mises en œuvre à Lorient au XVIII ${ }^{\mathrm{e}}$ siècle sont éparses dans les archives du Service historique de la Marine :

- par une requête des propriétaires d'un terrain situé près de Kernevel, on apprend l'ouverture d'une carrière dont les pierres ont été utilisées 
pour construire la redoute qui a été faite aux frais et par les ordres de la Compagnie des Indes;

- un document en date de 1761 fournit des précisions sur les journées d'ouvriers employés à remblayer deux carrières situées sur la " pointe de $\mathrm{K} / \mathrm{nel}$ " (Kernel), pouvant s'avérer dangereuses;

- les digues, évoquées plus haut, retenaient tout particulièrement l'attention des ingénieurs. Vers le milieu du XvIII ${ }^{\mathrm{e}}$ siècle, des travaux importants étaient entrepris pour la construction de telles digues autour des vasières du port. Vers 1760, l'ingénieur Saint-Pierre avait rédigé un mémoire sur les digues en pierres à exécuter en vue d'achever d'entourer les dépôts de vase à l'anse de Kergrois. La Compagnie des Indes avait alors demandé à l'ingénieur de préciser le lieu des carrières à exploiter dans ce but. En fait, la construction des digues soulevait deux problèmes : les approvisionnements en pierres, l'instabilité sur les vases...

- les environs de Lorient sont totalement dépourvus de schistes tégulaires. Dans ces conditions, les ardoises utilisées à Lorient au XVIII ${ }^{\mathrm{e}}$ siècle provenaient des environs de "Rhedon ", sans autres précisions - d'où elles pouvaient être acheminées par voie d'eau jusqu'à Lorient.

\section{En 1836, tentative d'établissement d'une liste des carrières du Morbihan}

Une autre source d'information sur les anciennes carrières ouvertes dans la région lorientaise, est disponible aux archives départementales du Morbihan ${ }^{13}$. Ce document se rapporte à la liste des carrières de ce département dressée en 1836. L'origine de ce répertoire est à rechercher dans la lettre adressée au préfet par le directeur général des Ponts et Chaussées le 11 mars de cette même année. En vue de faciliter la mission qui va être confiée à un ingénieur des Mines de dresser la carte géologique du département, " il serait fort utile (écrit le directeur) que l'ingénieur [...] chargé du travail ait à sa disposition, avant de commencer sa tournée géologique, un état général, faisant connaître, commune par commune, les carrières [...] qui sont ou qui ont été ouvertes dans le département ". Cette demande - adressée à toutes les préfectures - devait être transmise à M. M. les maires chargés d'y apporter " toute la célérité désirable ".

Cette enquête n'allait pas recevoir l'accueil espéré. C'est ce que laisse entendre une lettre du directeur général, en date du 22 juin 1836, qui « malgré [ses] demandes réitérées " n'a pu encore obtenir les renseignements nécessaires "pour la formation d'un tableau [...] indiquant par commune les carrières et exploitations de tous genres ". L'examen du répertoire concernant le Morbihan ne fait que confirmer ce regrettable état de fait. Un nombre impressionnant de communes n'ont fourni aucune information; d'autres se bornent à indiquer " point de carrière " alors qu'à l'évidence, il en existe. Les communes sont regroupées par arrondissement :

13. Arch. dép. du Morbihan S 501. 
- Ploermel. Sur 61 communes, deux seulement (Mauron et Ruffiac) ont fourni quelques indications, soit 1 sur 30 ;

- Pontivy. Sur 45 communes, 9 soit 1 sur 5 ;

- Vannes. Sur 74 communes, 24 réponses positives, soit 1 sur 3;

- Lorient. Sur 66 communes, 30, soit près de 1 sur 2, exception heureuse pour l'avancement de nos propos.

Ce faible pourcentage de réponses des maires à la demande de la préfecture ne laisse pas d'étonner. Plus que de négligence, faut-il y voir le manque d'intérêt des élus pour les ressources du sous-sol de leur commune, voire une certaine méfiance? Cette dernière hypothèse est confirmée par une lettre d'A. Paillette - qui avait été sous-directeur des mines de PoullaouenHuelgoat pendant six ans - adressée le 13 octobre 1835 au sous-préfet de Châteaulin ${ }^{14}$ : "La méfiance que les géologues excitent dans [ces] sauvages contrées [est] partagée à la fois par les pauvres paysans, par les maires ou leurs adjoints. " Et Paillette d'expliciter sa pensée : "Un géologue cassant des pierres, à droite, à gauche, leur semble un être tellement bizarre, qu'ils le considèrent au moins comme simplet, sinon comme fou. Aussi, ne se donnent-ils presque jamais la peine de répondre, lorsqu'il leur demande le nom du village, d'une carrière, d'une butte élevée, à forme remarquable ou d'une vaste lande ${ }^{15}$."

Comme cela a été signalé plus haut, l'arrondissement de Lorient fait exception par le nombre relativement élevé des réponses transmises à la préfecture. Dans ce qui suit, on se limite aux communes de la région lorientaise seules envisagées dans cet article (figure 1).

Les données fournies diffèrent beaucoup selon les communes. Surprenant, le cas de Ploemeur où de nombreuses carrières sont ouvertes au bord de la mer et qui n'a pas daigné répondre; plus étonnant encore le silence de Lorient... La réponse d'Hennebont s'avère par trop laconique : "Plusieurs carrières de grain et de moellons en exploitation ${ }^{16}$ ". Caudan n'apporte guère de précisions, se contentant de signaler " 13 carrières de moellons "; mais ce nombre, à lui seul, est fort instructif : il sous-entend que, dans le passé, pratiquement toutes les communes en Bretagne possédaient généralement plusieurs carrières, souvent ouvertes seulement à l'occasion des besoins. Dans ces conditions, la réponse des communes indiquant " point de carrière " ne correspond certainement pas à la réalité. Il est très rare que la nature de la roche exploitée soit spécifiée; le cas de " grain " (granite) est, en fait, assez peu fréquent : "à Quéven, plusieurs carrières de grain ". Les réponses se bornent généralement à dire s'il s'agit de moellons.

14. Arch. dép. du Finistère 8 S 3.

15. Ces annotations qui concernent le centre du Finistère s'appliquent également aux deux départements voisins.

16. Le terme " grain " cité à différentes reprises est celui employé naguère pour désigner le granite, appelé aussi " pierre de grain ". 


\section{Figure 1 - Localisation des carrières des environs de Lorient citées dans la liste établie en 1836 (Arch. départ. Morbihan S 501)}

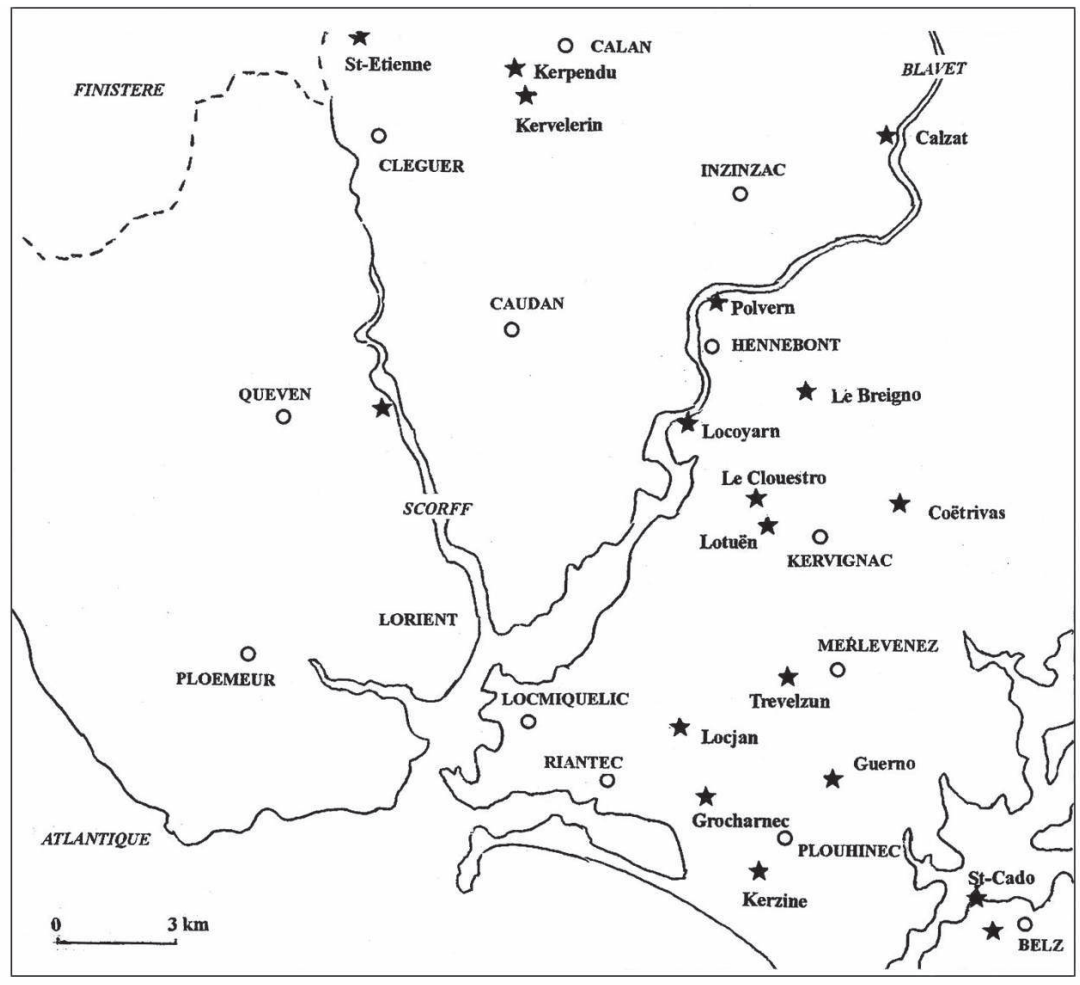

Quelques communes localisent leurs carrières avec une certaine précision, donnant, dans chaque cas, le nom du village où elles sont ouvertes. Ainsi, à Merlevenez sont indiquées deux carrières " de peu d'importance " à Trevelzun. À Belz, les carrières sont signalées près du bourg et à SaintCado; à Plouhinec, au Guerno, au passage d'Etel, à Kerzine; à Kervignac sont recensées 9 carrières : à la montagne de Coëtrivas ${ }^{17}$, à Clouestro, dans une lande près du bourg, à Lotuën, à la montagne du Breigno... À cette date, Locmiquélic où sont indiquées 4 carrières, dépend de la commune de Riantec. Cléguer signale plusieurs carrières en précisant leur état actuel : celle de Saint-Etienne n'est ouverte que depuis deux ans; celle de Kervelerin est indiquée comme étant en exploitation.

Le cas de Guidel est un peu exceptionnel car il ne s'agit pas de carrières livrant des matériaux de construction, mais du minerai de fer; elles ont " cessé d'être exploitées depuis 1834 ". La carrière de Calzat en Inzinzac

17. Le mot " montagne ", en Bretagne, indique le plus souvent un faible relief dans une topographie aplanie. 
ouverte sur le versant du Blavet constitue également un cas spécial; le texte précise qu'il s'agit d'une " carrière de qualité de pierres ou cailloux blancs imitant un peu le marbre; très propre à la fabrication du verre ». Il s'agit, en fait, d'un filon de quartz très pur.

\section{Autres sources d'informations vers le milieu du XIX ${ }^{\mathrm{e}}$ siècle}

S'il constitue incontestablement un document pour la localisation des anciennes carrières dans la région lorientaise, le répertoire de 1836 s'avère en fait, comme nous l'avons laissé entendre, très incomplet, d'où l'impérieuse nécessité d'avoir recours à d'autres sources d'informations pour cette période. L'un des meilleurs documents, publié en 1848, mais dont les enquêtes sur le terrain remontent à plusieurs années antérieurement - c'est-à-dire à la même période que le répertoire précité - est dû à Th. Lorieux et E. de Fourcy ${ }^{18}$; il apporte des précisons, parfois chiffrées, mais sur quelques carrières seulement :

- Granite de Belz-Crach. Cette pierre a été extraite en plusieurs points. Toutefois, les exploitations, ouvertes par intermittence, "ne fournissent que des matériaux médiocres par la qualité et par les dimensions ". Les auteurs font remarquer que, le plus souvent, les extractions n'étaient pas " à proprement parler, de véritables carrières. On se bornait à faire sauter çà et là, le long des bords de la mer ou sur ceux des rivières qui s'y rendent, les rochers susceptibles de donner, à proximité des points d'embarquement, des pierres saines ayant les dimensions demandées ". Les carrières des environs de Belz font cependant exception. "On en a tiré beaucoup de pierres d'appareil pour le phare de Belle-Île ${ }^{19}$ et pour les grands travaux de Lorient. " Les exploitations les plus importantes étaient situées " près du moulin des Oies (500 mètres à l'ouest de Belz), près de la chapelle de SaintCado, dans les îles et sur les bords de la rivière d'Etel ". Ces carrières sont, à cette date, les seules de ce massif granitique qui aient encore quelque importance " par suite de leur voisinage du port de Lorient".

- Granite de Ploemeur. Près du château de Soy entre Lorient et Ploemeur est exploité alors un granite fissuré fournissant des moellons. La production annuelle est de l'ordre de $15000 \mathrm{~F}$. Le granite est également extrait sur le bord de la mer, près de Saint-Jude; la pierre livrée est peu tenace.

- Granite d'Hennebont. Les carrières sont ouvertes en bordure du Blavet, en amont d'Hennebont près de Polvern. Contrairement au granite de Ploemeur, la pierre offre une bonne résistance, d'où son emploi pour les grands travaux de Lorient.

Dans leur conclusion sur l'exploitation des granites, les deux ingénieurs constatent l'étendue des massifs, mais regrettent qu'il soit " si difficile d'y trouver des pierres de grande dimension ». Ils ajoutent que " cette difficulté

18. LoRIEUX Théodore, Fourcy Eugène de, Carte géologique du Morbihan, op. cit.

19. Il s'agit du grand phare de Goulphar allumé en 1836. 
tient à ce qu'aucune carrière n'est exploitée régulièrement : on se contente d'extraire à la surface du sol, les blocs dont on a momentanément besoin ". D'où leur souhait de voir l'Administration faire " ouvrir quelques carrières dans les points les plus convenablement situés pour les transports ». Dans les devis, il serait alors prescrit " aux entrepreneurs de travaux publics d'y faire leurs approvisionnements ${ }^{20} "$.

\section{Sources diverses}

Plusieurs autres sources de données sur les anciennes carrières peuvent encore être signalées :

- les cartes géologiques au 1/80000 feuilles "Lorient " (1885) et "Vannes " (1890) dues à Ch. Barrois, localisent quelques carrières en précisant s'il s'agit de pierres de taille ou de moellons, voire de matériaux d'empierrement. Le fond géologique en couleurs de la carte indique ipso facto la nature de la roche exploitée. En fait, peu de carrières sont reportées;

- les archives départementales du Morbihan et du Finistère - essentiellement dans la série S (concernant les Travaux publics : phares, canaux, ports...) possèdent des informations sur plusieurs carrières exploitées ou tout au moins prévues dans les devis;

- l'examen stéréoscopique des photographies aériennes de l'IGN ${ }^{21}$ permet de localiser avec une grande précision de nombreuses carrières depuis longtemps abandonnées, à condition toutefois qu'elles n'aient pas été entièrement comblées. Les récentes cartes au 1/25000 du même organisme indiquent aussi la position de ces anciennes carrières ${ }^{22}$.

Ces diverses informations doivent être impérativement complétées par des investigations sur le terrain.

À l'aide de toutes les sources succinctement évoquées, il va être tenté maintenant de présenter une vue plus détaillée des anciennes carrières de la région lorientaise. Bien que ce travail de longue haleine ne soit pas encore totalement achevé, il a paru, vu l'ampleur des données déjà obtenues, de le publier sans plus attendre. Toutefois, en vue d'éviter des répétitions risquant de devenir fastidieuses, un choix se devait d'être effectué. Les sites décrits sont regroupés de la manière suivante : carrières littorales (Ploemeur); carrières sur les versants des rias (le Scorff, le Blavet, Rivère d'Etel); carrières dans les terres.

20. Lorieux et de Fourcy signalent le quartz de Kernacouet sur la rive droite du Blavet. Ce filon de quartz blanc, naguère exploité pour la verrerie de Kernevel près de Lorient est, à l'évidence, désigné ultérieurement sur le nom de Calzat.

21. Institut géographique national.

22. Et évidemment la localisation des carrières actuellement en activité. 


\section{En bordure de l'Océan}

Le massif leucogranitique de Ploemeur s'étend d'ouest en est sur une quinzaine de kilomètres entre Fort-Bloqué et la Petite Mer de Gâvres; au nord, il est intrusif dans des micaschistes; à l'est, il recoupe le granite de Belz-Crach; ses limites ouest et sud sont masquées par l'Atlantique. Pétrographiquement, comme son nom l'indique, il s'agit d'un leucogranite blanchâtre, le plus souvent à grain moyen, à quartz globuleux riche en muscovite (mica blanc) à reflet argenté. Généralement à texture équante, il peut présenter localement une légère orientation. En surface, il a tendance à devenir friable. Il est fréquemment recoupé par des filons aplito-pegmatitiques $^{23}$ à tourmaline noire. Après sa mise en place, il a subi, à la faveur de fracturations transversales, des processus d'altération hydrothermale conduisant à la formation de gisements de kaolin ${ }^{24}$.

La proximité de Lorient a eu certainement un impact majeur sur le développement des carrières. Si quelques sites d'extraction étaient ouverts à l'intérieur des terres (Soy, Kervinio), la plupart des exploitations s'échelonnaient sur le littoral; en dépit de leur abandon, elles demeurent encore facilement identifiables (figure 2) :

- à proximité immédiate de Fort-Bloqué et tout particulièrement au sud et à l'ouest, les extractions ont été intenses sur l'estran, comme l'attestent l'attaque d'un récif devenu ainsi résiduel, les gradins de taille séparés par de larges surfaces subhorizontales, les trous de perforation verticaux à la barre à mine, les mares créées à l'emplacement des masses rocheuses excavées... Localement, les anciennes zones d'extraction sont recouvertes par un dense tapis de moules soulignant que, peu à peu, la Nature reprend ses droits;

- à quelque distance au sud de Fort-Bloqué, une carrière s'étire en bordure du littoral sur plus de 100 mètres; sa largeur qui atteint plusieurs dizaines de mètres, témoigne de l'important recul du trait de côte primitif. La disposition du leucogranite en dalles facilitait l'extraction. En avant de l'actuelle ligne de rivage, quelques points hauts, constituant à présent de gros rochers résiduels, ont été épargnés par les carriers, peut-être du fait de l'arrêt des travaux ou de la qualité moins bonne de la pierre? La sole irrégulière est accidentée de mares néo-formées et vers les terres, les fronts de taille ont façonné une falaise artificielle;

- au sud-sud-ouest du fort du Talud, les exploitations sont attestées par un vaste platier de néo-formation parsemé de mares artificielles et limité au nord par une basse falaise anthropique. L'irrégularité dudit platier suggère

23. Aplite = granite à grain très fin; dans les pegmatites, les cristaux sont souvent énormes.

24. Ici hors de notre propos. Les gisements de kaolin de Ploemeur sont les plus importants de France. Se reporter à CHAURIS, Louis, "Vue d'ensemble sur les occurrences de kaolin d'origine hydrothermale en Bretagne (France) ", Bull. Soc. Sc. nat. Ouest de la France, nelle série, 18, (4), 1996, p. 157-188. Lire aussi BEAUREGARD Bruno de, « Les kaolins de Ploemeur en Morbihan ", Bull. Association bretonne, cXVI, 2007, p. 257-278. 
l'avancement quelque peu anarchique des extractions, contrôlées par la structure en bancs peu inclinés du leucogranite et l'orientation des diaclases (ou cassures naturelles). Vers le large, une barrière rocheuse, non exploitée, protège ainsi plus ou moins les chantiers d'abattage. Les galets qui ont envahi la sole de la carrière proviennent en partie du remaniement, par les vagues, des fragments de taille;

- parmi les autres sites d'extraction : au nord de l'anse de Courégant, une carrière très allongée s'avançant dans les terres; au nord-ouest de Kerroc'h, des deux côtés d'une crique, depuis l'estran jusqu'au sommet de la petite falaise; près de la cale de Lomener; à Port-Louis, plusieurs points sur l'estran au sud-ouest des remparts, ainsi qu'à l'île aux Souris avec un haut front de taille vertical; près du fort de Port Puns à Gâvres ${ }^{25}$.

\section{Figure 2 - Anciennes carrières ouvertes dans le leucogranite de Ploemeur (prospections de l'auteur)}

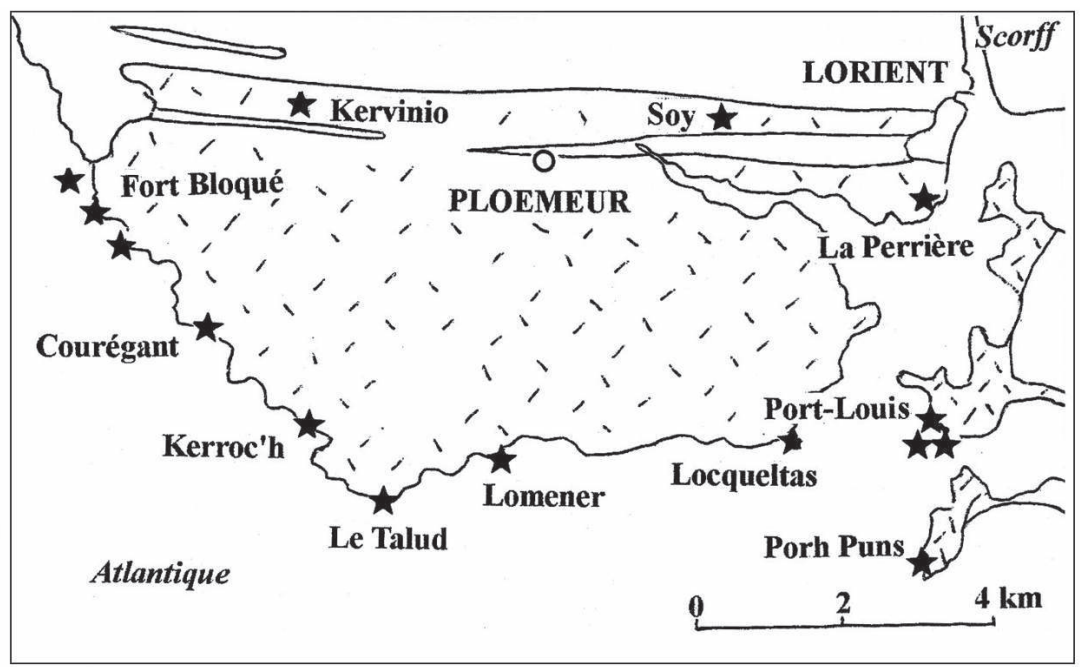

Au total, les sites d'extraction dans le leucogranite de Ploemeur fournissent d'excellents exemples de carrières sur le littoral lorientais. Ces exploitations ont modifié durablement l'environnement côtier en développant une morphologie d'érosion anthropique originale (platiers creusés de mares; pitons artificiels; gradins de taille; néo-falaises...), mais aussi en participant aux processus de sédimentation par l'abandon aux vagues d'innombrables débris de taille qui acquièrent progressivement l'émoussé

25. Pour plus de détails voir CHAuRIS, Louis, « Exploitation et mise en œuvre du leucogranite de Ploemeur ", Pierre Actual, n 870,2009 , p. 72-79. 
des galets... L'engouement prolongé pour ces modalités d'exploitation s'explique par deux raisons commandées par la mer : ici, la roche, débarrassée par les eaux de son manteau d'altérites qui recouvrent souvent les sites terrestres, était directement exploitable, sans coûteux travaux de " découverte "; mieux, aux époques où les charrois s'avéraient pénibles et dispendieux, les lourds matériaux extraits pouvaient être acheminés par voie d'eau. Ainsi s'interprètent à la fois la diffusion et la mise en œuvre du leucogranite de Ploemeur dans toute la région lorientaise.

\section{En bordure du Scorff}

Les informations sur les anciennes extractions de granite à proximité du Scorff sont peu nombreuses et surtout peu explicites. H. F. Buffet rappelle que lors des travaux commencés à l'Orient en 1732 par la Compagnie des Indes, " des carrières furent choisies en bordure du Scorff dont la pierre à fond gris de lin était réputée ". À cette date, une cinquantaine d'ouvriers travaillaient dans les perrières " à tailler les blocs de granite ". L'auteur ajoute qu' «ils étaient consciencieux, mais ne se pressaient guère ». En 1741, l'ingénieur Saint-Pierre (infra) cite une carrière à Sacquevin (Sac'h Quéven); une autre carrière est située à un quart de lieue en amont; une troisième nettement plus en aval près de Querantrey (Kerentrech). En 1836, dans la liste des carrières du Morbihan, il est seulement indiqué (infra) la présence à Quéven, commune riveraine du Scorff, de " plusieurs carrières de grain ". Dans le supplément au Dictionnaire d'Ogée, paru en 1843, il est noté, à l'article "Quéven ", que "plusieurs carrières de pierres de taille [...] ont fourni la majeure partie des matériaux employés au bassin et aux cales [...] de Lorient. Sur la carte géologique de 1885, aucune carrière n'est figurée en bordure du Scorff".

En fait, l'étude entreprise sur le terrain suite à l'examen des photographies aériennes et de la carte au 1/25000 de l'IGN, permet d'établir que les sites d'extraction ont été naguère relativement nombreux ici (figure 3 ). Si la carrière de Kerentrec'h est aujourd'hui comblée par suite des progrès de l'urbanisation dans la partie nord de Lorient, si la carrière de Sac'h Quéven n'a pu être décelée, plusieurs autres carrières sont encore visibles à proximité du Scorff : d'une part, sur la rive droite, en Quéven, à environ un kilomètre au nord-nord-ouest de Sac'h-Quéven et au nord du lieu-dit " Le Roze ", dans des bois; d'autre part, sur la rive gauche, à quelque distance de la rivière, en Caudan, près de Kerviec et de Pendreff.

\section{Sur les bords du Blavet}

À l'inverse du district précédent (rives du Scorff), les informations sont nombreuses et précises sur les anciennes carrières de granite ouvertes en bordure du Blavet au nord d'Hennebont près de Polvern (figure 4). Les extractions sont anciennes ainsi que l'atteste l'emploi de cette roche 
pour l'église de cette cité au XVI ${ }^{\mathrm{e}}$ siècle, et prolongées puisqu'elles se poursuivent encore au $\mathrm{xx}^{\mathrm{e}}$ siècle. Ces exploitations sont mentionnées dans le supplément au Dictionnaire d'Ogée (1843) signalant la présence de "carrières de quelque importance près de l'ancienne abbaye de La Joie ". Lorieux et de Fourcy (1848) soulignent que " la roche se taille bien et est d'une bonne résistance; aussi les carrières y sont-elles nombreuses et d'une exploitation assez suivie "; à cette époque, elles occupent 20 à 30 ouvriers, livrant du granite à 14/15 F le mètre cube; la valeur de la production est estimée à $18000 \mathrm{~F}$ (soit environ $1200 \mathrm{~m}^{3} / \mathrm{an}$, en pavés et pierres de construction). Le Répertoire de $1889^{26}$ indique le nom des exploitants (Audo, Cottenseau), le prix du $\mathrm{m}^{3}$ sur carrière $(44 \mathrm{~F})$ et à la gare ou au port (50 à $55 \mathrm{~F}$ ); il souligne l'importance de la masse compacte exploitée sur 20 à 24 mètres de profondeur. Le granite de Polvern est à grain fin, à biotite (mica noir), parsemé de quelques rares feldspaths blanchâtres de plus forte dimension; il est massif, très dur, à texture équante, offrant diverses teintes dans les gris, parfois à légère nuance bleutée.

De précieuses informations sur les carrières de Polvern sont fournies dans le rapport présenté lors du projet de construction du phare d'Eckmühl à Penmarc'h (Finistère) ${ }^{27}$. Dans ce projet, seul le soubassement du phare était prévu en kersanton de la rade de Brest; le parement du fût de la tour et l'escalier devaient être exécutés avec le granite des carrières de Polvern ${ }^{28}$. Mais voici que l'entrepreneur Vabre, adjudicataire, émet des doutes sur

26. Répertoire des carrières de pierre de taille exploitées en 1889. Librairie polytechnique Baudry et Cie, édit. Paris, 1890, 322 p.

27. Arch. dép. du Finistère 4 S 1274.

28. Curieusement orthographiée Polhuern. 


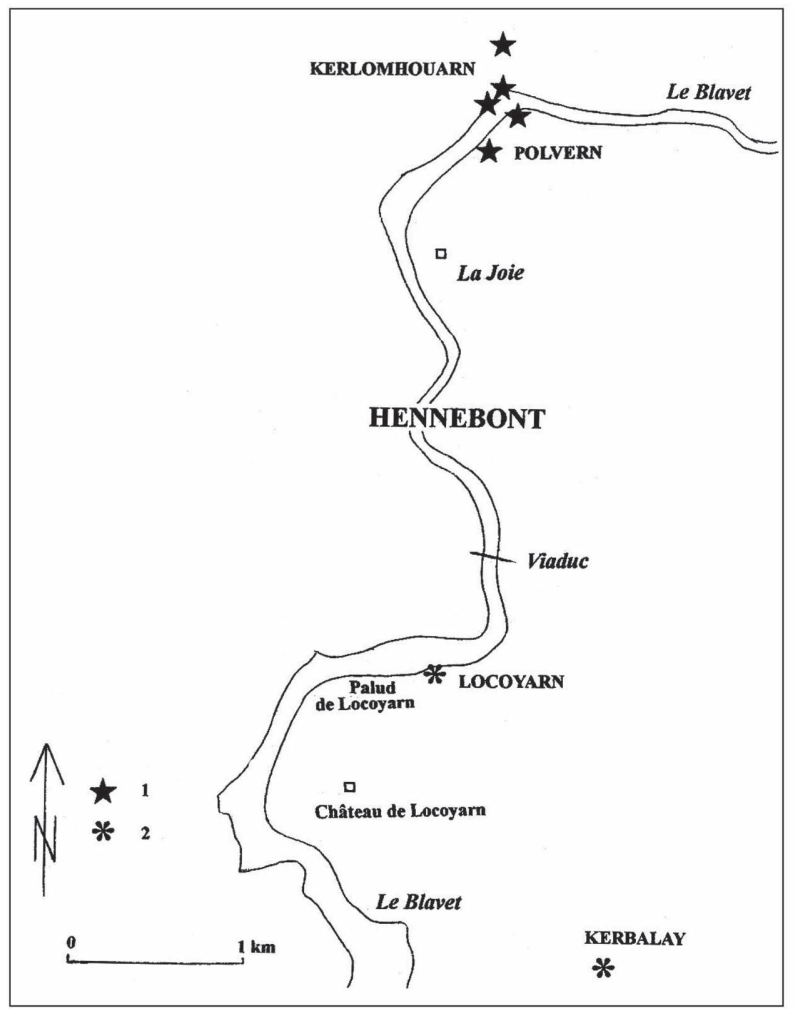

Figure 4 -

Localisation des anciennes carrières ouvertes sur les versants du Blavet de part et d'autre d'Hennebont (prospections de l'auteur)

l'aptitude de ces exploitations à livrer les volumes exigés. Tel n'est pas l'avis de l'ingénieur ordinaire des Ponts et Chaussées, Duperrier qui, dans son rapport du 4 novembre 1893, s'efforce de réfuter la proposition de Vabre demandant un changement de carrière. Sur la rive gauche du Blavet, à environ trois kilomètres au nord d'Hennebont, écrit l'ingénieur, les carrières sont au nombre de cinq : elles présentent " des fronts d'attaque de 12 à 18 mètres de haut, et de 8 à 20 mètres de large [...]. Trois au moins [...] peuvent fournir le granit [sic] bleu à grain fin " exigé au devis. Il reconnaît toutefois que leur " exploitation méthodique [...] est abandonnée depuis 1860 environ ", se limitant " à des fournitures de pavés et de bordures de trottoirs pour la ville de Lorient. Aucune carrière ne compte plus de 5 ouvriers ". Cependant, l'un des exploitants s'engage à fournir, avec l'aide de ses voisins, $350 \mathrm{~m}^{3}$ en un an, ce qui permettrait d'obtenir le volume demandé en moins de deux ans. L'ingénieur ne doute pas que " ce chiffre ne soit dépassé, si l'on veut, dès maintenant, reprendre l'exploitation dans les mêmes conditions qu'il y a quarante ans "; il rappelle que dans la première partie du XIX ${ }^{\mathrm{e}}$ siècle, les carrières de Polvern ont été « le centre d'une industrie considérable [...]. Lors de la construction des bassins de radoub à Lorient, il y avait plus de 200 ouvriers ». Le débit mensuel atteignait alors $100 \mathrm{~m}^{3}$. 
Par ailleurs, toujours selon l'entrepreneur Vabre, Polvern ne pourrait livrer des pierres de grand appareil, affirmation que conteste également l'ingénieur Duperrier, en indiquant qu' « un bloc [fendu] a donné deux morceaux de 1,50 m de long, 0,90 de large et 0,60 m d'épaisseur chacun ». En fait, il semblerait que ce soient les difficultés de remise en état du site de Polvern qui rebutent l'entrepreneur, ce qu'attestent " les amas d'attraits qui encombrent la partie inférieure des bancs ". Le rapport de l'ingénieur contient également d'autres données intéressantes sur les modalités de l'exploitation des carrières de Polvern et les qualités exceptionnelles de ses pierres. Les carriers n'osent s'engager à assurer un débit régulier par mois et, pour la raison suivante : "Il se présente quelquefois (disent-ils) dans un banc, une couche de granit gris [...] dont l'extraction dure [parfois] un mois, mais on retombe presque toujours sur de la pierre bleue. "Ce à quoi l'ingénieur objecte qu'en mettant « 10, 15, 20 hommes ou plus dans chaque carrière [...], il est probable qu'on ne resterait plus d'un mois dans les bancs de pierre grise ". Les comparaisons faites par l'ingénieur avec d'autres carrières proposées par l'entrepreneur ne font que démontrer les qualités du granite de Polvern. En voici deux exemples. Pont-Aven ${ }^{29}$ " est une belle pierre [...] résistante, susceptible de ciselures très nette, mais à gros grain [...]; elle est bien moins dure que le Polhuern et moins fine d'aspect ". La pierre de " l'Île Grande ${ }^{30}$ n'a pas la couleur, ni la texture du granite de Polhuern, il s'harmoniserait mal [...] avec le kersanton du soubassement ". Et l'ingénieur de conclure : "De l'aveu des gens de métier, la pierre de Polhuern passe pour le plus dur des granites [...]; il pourrait y avoir un inconvénient très réel à l'abandonner. " Mais alors comment expliquer pourquoi les carrières de Polvern ont-elles vu décroître si nettement leur activité? Selon l'ingénieur Duperrier, l'exploitation des carrières de Trégunc-Pont-Aven et de Scaër " a fait beaucoup de tort à celles de Polhuern [...]; dès que les travaux de grande importance ont été terminés, (ces pierres plus tendres) ont eu la préférence sur les matériaux durs et difficiles à tailler d'Hennebont ". Mais d'ajouter aussitôt que c'est pour cette raison de dureté que Polvern avait été désigné au devis du phare d'Eckmühl ${ }^{31}$.

Ultérieurement, du fait justement de ces qualités exceptionnelles de dureté et de résistance, les carrières de Polvern allaient, dans les années 1920, prendre une ampleur jusqu'alors inégalée, sous l'impulsion de la "Société des Carrières et Travaux de Paris ", avec la fabrication de pavés. Le choix de Polvern était basé sur des analyses comparatives effectuées par le laboratoire de la Ville de Paris en 1913, à partir de 16 gisements.

29. Le granite de Pont-Aven est plus connu sous l'appellation de Trégunc. Voir CHAURIS, Louis, "Les multiples facettes du granite de Trégunc ", Courrier du Léon/Progrès de Cornouaille, 17 juillet, 21 août, 11 et 25 septembre 1993.

30. ChaurIs, Louis, " Carrières du bord de la mer. Ile Grande et îlots voisins (Côtes-duNord) ", $115^{\mathrm{e}}$ Congrès nat. Soc. sav. Avignon, Colloque "Carrières et constructions ", 1991, p. $305-321$.

31. Finalement, le granite de Polvern allait être délaissé au profit du kersanton. 
L'extraction fait appel à un matériel de perforation et de débitage à air comprimé. La manutention des blocs s'exécute avec l'aide de chariots sur des voies Decauville. L'acheminement des matériaux jusqu'aux ateliers est assuré par des wagonnets attelés à de locotracteurs. L'énergie électrique alimente les appareils de façonnage des pavés. En 1927, avec environ 200 ouvriers - dont une majorité d'étrangers (Italiens, Suédois, Norvégiens, spécialistes de la taille du pavé) ${ }^{32}$ - la production devait atteindre annuellement environ 30000 tonnes. La pierre est expédiée par le Blavet canalisé (relié au canal de Nantes à Brest) ou par voie maritime. Des remorqueurs entraînent des chalands de 120 tonnes en poids lourd. Dès 1921, le site est desservi par le rail, grâce à un embranchement particulier. Pendant la seconde Guerre mondiale, les carrières de Polvern ont fourni une contribution significative à la construction du Mur de l'Atlantique, avec des expéditions par trains et par péniches.

Plusieurs autres carrières étaient ouvertes sur la rive gauche du Blavet, en face des exploitations de Polvern, près de Kerlomhouarn; aujourd'hui abandonnées, elles fournissaient aussi un granite gris à grain fin, avec quelques feldspaths blancs de granulométrie supérieure.

Un granite totalement différent du granite de Polvern a été exploité dans le passé - dès le Moyen Age ainsi que l'attestent les remparts d'Hennebont - en aval de cette cité, près du lieu-dit Locoyarn en bordure du Blavet qui permettait d'acheminer la production par voie d'eau (figure 5). Ce granite était extrait aussi, dans les terres, près de Kerbalay; à l'évidence, plusieurs perrières sont aujourd'hui comblées. La roche est facilement identifiable par l'étonnante abondance de cristaux feldspathiques blanchâtres, très allongés, pluricentimétriques, mais de faible épaisseur, disposés plus ou moins parallèlement les uns aux autres. Cette belle pierre dont on regrette l'abandon a été surtout exploitée du XVI ${ }^{\mathrm{e}}$ au XVIII ${ }^{\mathrm{e}}$ siècles $^{33}$.

\section{À proximité de la rivière d'Etel}

Le massif granitique de Belz-Crach s'étend sur près de $30 \mathrm{~km}$ entre le Blavet et la rivière d'Auray. Masqué au sud sous l'Atlantique, il est intrusif à l'est et au nord dans des complexes métamorphiques; à l'ouest, il est recoupé par le leucogranite de Ploemeur. La roche, à grain fin, très légèrement porphyroïde, à biotite et rare muscovite, offre une teinte grisâtre à nuance bleutée.

Dans le passé, les sites d'extraction ont été nombreux (supra: archives départementales du Morbihan série S 501; Lorieux et de Fourcy, 1848), mais généralement de faible importance individuelle. Comme l'atteste une lettre en date du 2 avril 1928, en réponse à l'appel d'offre pour la fourniture

32. Gullchet, Jacques, Hennebont. De l'Empire à la Deuxième Guerre mondiale, Hennebont, Impr. Le Roux, 1987.

33. CHAURIS, Louis, Mise en œeuvre pluriséculaire du granite de Locoyarn, une pierre méconnue du Morbihan (sous presse). 
de granite taillé nécessaire à la construction de l'Ecole navale à Brest ${ }^{34}$, la situation s'est, à cette date, sensiblement modifiée. Les carrières de l'entreprise Jamet et Fils ouvertes près de Belz, disposent alors d'un " outillage des plus modernes, air comprimé, derrick [...] permettant [...] d'assurer un débit mensuel important pouvant aller jusqu'à 300 et $400 \mathrm{~m}^{3}$ de granit taillé ". L'entrepreneur fait observer que son personnel "très expérimenté se charge de toutes les tailles et sculptures du granit ". Il indique que la pierre proposée est " un granit bleu, dur [...] se polissant très bien, inaltérable [...], se présentant en carrière sous forme de blocs de toutes longueurs et de toutes épaisseurs". Il précise qu'il est possible de débiter des éléments atteignant $3 \mathrm{~m} \mathrm{x} 3 \mathrm{~m}$ x $3 \mathrm{~m}$, c'est-à-dire de près de $30 \mathrm{~m}^{3} \mathrm{La}$ situation de ses exploitations à deux kilomètres du port d'Etel permet un chargement du granite sur bateau et par suite un acheminement au loin. Aujourd'hui, toutes les exploitations ont cessé.

\section{Dans les terres}

Un dernier ensemble de carrières était situé au nord de Lorient dans des roches écrasées en liaison avec un des accidents tectoniques majeurs du Massif armoricain, connu sous le nom de "zone broyée sud-armoricaine $^{35}$ ". Toutes les exploitations sont aujourd'hui abandonnées, exceptée l'une d'elles, ouverte en 1996 près de Coët-Loc'h (à proximité d'une carrière noyée), appartenant au groupe " Eurovia-Vinci " et produisant des granulats à partir d'un orthogneiss (ancien granite) mylonitisé. Les carrières abandonnées exploitaient des mylonites (roches intensément écrasées) pour l'empierrement : Coët-Loc'h; Bonne-Nouvelle, non loin du Blavet, montrant encore un impressionnant front de taille (au moins cinq gradins, sans compter les parties inférieures noyées et par suite invisibles); La Villeneuve, s'étirant sur environ 500 mètres de long selon la direction de la mylonitisation. La carrière de Kergueris au sud-est de Calan, ouverte dans des orthogneiss mylonitiques, livrait de beaux moellons plats et des dalles de diverses teintes, utilisées jusqu'à une date récente dans toute la région lorientaise ${ }^{36}$.

Dans le même contexte géologique, la carrière de Calzat occupe une place un peu à part. Elle était ouverte dans le puissant filon quartzeux qui jalonne le linéament sud-armoricain, fournissant un superbe granulat éclatant de blancheur et d'une pureté exceptionnelle, utilisé en agglomérés pour parements. Antérieurement, ce filon de quartz était exploité en différents points pour l'empierrement des routes. Dans le passé, le quartz, par suite de sa dureté et de son inaltérabilité, constituait un matériau de choix

34. Archives de l'Ecole navale à Brest. Voir CHAURIs, Louis, " Quels granites pour l'École navale? ", Les Cahiers de l'Iroise, 157, 1993, p. 13-18.

35. CHAURIs, Louis, "Observations minéralogiques et chimiques sur l'évolution hydrothermale ultime du linéament sud-armoricain en Bretagne ", Bull. Soc. Sc. nat. Ouest de la France, ${ }^{\text {elle }}$ série, t. 34, (1), 2012, p. 52-58.

36. Par exemple au Palais des Congrès à Lorient. 
Figure 5 - Carrières le long de la zone broyée sud-armoricaine. 1- Orthogneiss mylonitique de Kergueris fournissant moellons et dalles. 2-Mylonites livrant des granulats. 3- Quartz. Seule la carrière de CoëtLoc'h est à présent en activité. (Prospections de l'auteur)

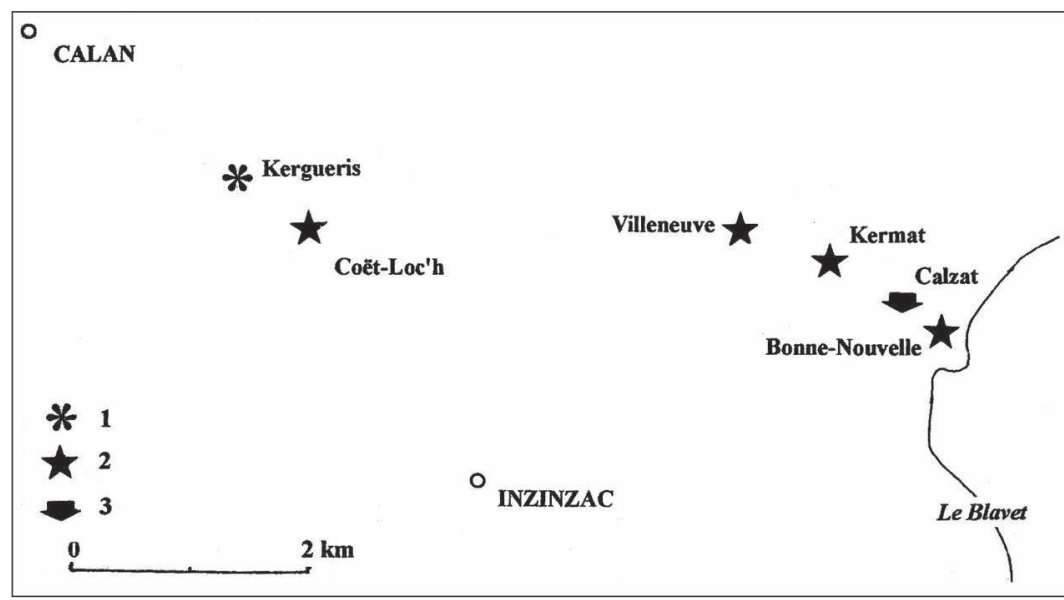

pour l'entretien des chemins. Les ingénieurs de Ponts et Chaussées manifestaient un réel engouement pour cette roche qui, à cet égard, surpassait toutes les autres. À présent, avec la circulation automobile sur pneumatique, ce matériau est abandonné pour la raison même qui le faisait naguère si recherché, à savoir sa dureté.

\section{Conclusions}

À l'issue de ces analyses sur les anciennes carrières de la région lorientaise, plusieurs aspects de portée plus générale peuvent être synthétisés.

\section{Nature des roches exploitées : un large éventail}

Dans une très forte proportion, il s'agit de granites. Toutefois, il s'avère indispensable d'être plus précis. Dans la région lorientaise, les intrusions granitiques appartiennent à des types différents : granite gris ou bleuté à grain fin de Polvern; granite porphyroïde blanchâtre de Locoyarn; granite gris-bleuté à grain fin de Belz; leucogranite - ou granite clair, riche en muscovite - de Ploemeur. Les autres roches (orthogneiss, mylonites, quartz...) ont été nettement moins recherchées pour les constructions. Par suite de cette diversité, les carrières pouvaient livrer, selon les sites, des pierres de taille, des moellons, des pavés, des matériaux d'empierrement; les entrepreneurs disposaient ainsi d'un large choix. 


\section{Appel à des carrières distales : une nécessité}

Cependant, en dépit de leur diversité, les anciennes carrières de la région lorientaise n'ont pu fournir toutes les pierres nécessaires au développement de la grande cité maritime. Errer dans Lorient révèle très vite qu'appel a été fait, déjà dans le passé et plus encore à présent, à des roches lointaines. Les unes proviennent de Bretagne : naguère, le granite de Trégunc recherché pour les infrastructures maritimes mais aussi pour l'habitat, du fait de son aptitude à procurer des éléments de grand appareil ; le kersanton des confins orientaux de la rade de Brest, non seulement pour la statuaire (monument aux morts) mais aussi pour les Travaux publics (viaduc du chemin de fer sur le Scorff)...; récemment, granites du Hinglé et de Ploumanac'h dans les Côtes-d'Armor, du Huelgoat dans le Finistère, de Bignan dans le Morbihan... Les autres, extraites hors des limites du Massif armoricain, ont été recherchées dès les débuts de Lorient au XVIII ${ }^{\mathrm{e}}$ siècle, entre autres le tuffeau du Val de Loire et le calcaire des Charentes. La mise en œuvre à Lorient et ses abords à la fois de roches proximales et distales confère aux constructions un polylithisme écartant, agréablement, le monotonie qui eût été liée à une seule source d'approvisionnement.

\section{Localisation des sites d'extraction : les atouts de l'eau}

Leur répartition est le reflet de la position géographique de Lorient permettant trois modalités différentes d'extraction. Au bord de l'océan, avec deux atouts majeurs : exploitation directe de la roche débarrassée de ses altérites par la mer et acheminement aisé par voie d'eau. Sur les versants de deux importantes rias, le Scorff et le Blavet, débouchant dans la rade de Lorient, avec ici encore, l'avantage de la navigabilité; plus éloigné, la rivière d'Etel offrait également cet atout. À l'intérieur des terres, les carrières étaient dépourvues de ces avantages, aussi étaient-elles nettement moins recherchées.

\section{État actuel des carrières : un abandon généralisé}

Comme partout en Bretagne, ici aussi, d'innombrables sites d'extraction sont aujourd'hui abandonnés et l'un des objectifs de cet article a été de les sortir de l'oubli : leur litanie s'échelonne un peu comme un glas... Si on ne savait que les causes de ces abandons sont multiples et complexes, on en viendrait à se demander si la Bretagne n'a pas été victime de sa trop grande richesse en granites pour les délaisser ainsi... Les modalités consécutives à ces abandons sont variées. Souvent, les carrières ont disparu entièrement par comblement et seule la toponymie, parfois, en conserve encore le souvenir : tel est le cas de "La Perrière " à Lorient. D'autres sites ont été transformés en décharge : l'immense carrière de Kermat près d'Inzinzac s'est muée en centre de stockage pour déchets ménagers de la région lorientaise; son accès est interdit sans autorisation. Certaines excavations 
sont partiellement noyées, telle "Bonne-Nouvelle ". Fréquemment, après l'arrêt des extractions, les carrières sont progressivement envahies par la végétation, avec, toutefois, des statuts divers : des deux immenses carrières ouvertes sur la rive gauche du Blavet, de part et d'autre de l'écluse de Polvern, la carrière " aval " a été nettoyée et laisse percevoir un front de taille subvertical où se distinguent très bien les trous de perforation à la barre à mine. La carrière de Locoyarn au sud d'Hennebont a été aménagée pour " les Gens du Voyage "... En fait, seules les carrières littorales sur la côte de Ploemeur sont restées comme figées depuis l'arrêt des extractions et seul le va-et-vient des flots adoucit lentement les platiers anthropiques.

\section{Patrimoine industriel : des sites à aménager}

Les carrières font partie intégrante du patrimoine, plus précisément du domaine silencieux de l'archéologie industrielle ${ }^{37}$. Dans ces conditions, ne serait-il pas souhaitable que quelques unes d'entre elles soient réaménagées dans un souci bien compris de l'environnement, avec panneaux explicatifs précisant l'histoire du site. Le cas de la célèbre carrière de Polvern citée à l'instant se prêterait sans doute bien à cette "mutation ", après vérification de l'état des fronts de taille soumis aux aléas des risques d'éboulements ${ }^{38}$. Alors l'ancienne carrière, comme transfigurée, retrouverait-elle une seconde vie.

37. Consulter sur ce sujet l'excellent numéro des Arts de l'Ouest: Archéologie industrielle en Bretagne, PUR, 1991, 290 p., où sont présentés de nombreux exemples.

38. Un exemple remarquable : l'aménagement de l'ancienne carrière de Lemptegy ouverte dans un volcan de la chaîne des Puys. 
Photo 1 - L'extraction du granite de Ploemeur, près de Fort-Bloqué, était facilité par sa structure en dalles subhorizontales. Au premier plan, platier de néo-formation parsemé de mares artificielles. Au fond, piton résiduel épargné par l'exploitation (L. Chauris)

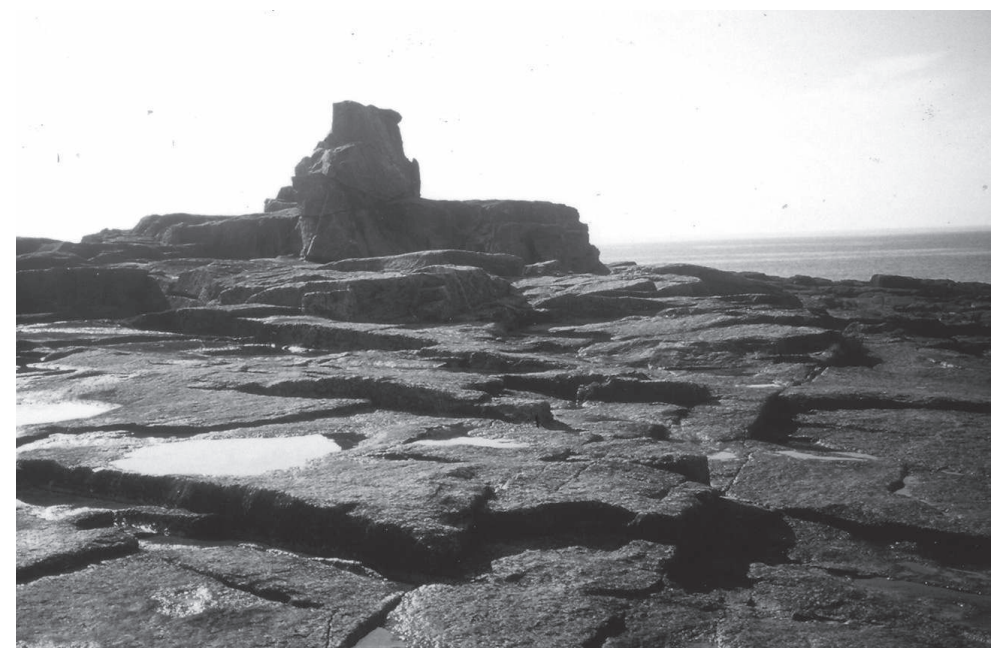

Photo 2 - Près de Fort-Bloqué, exploitation du granite de Ploemeur par la méthode des grands trous de perforation à la barre à mine. Au premier plan, mare formée à l'emplacement des blocs excavés (L. Chauris)

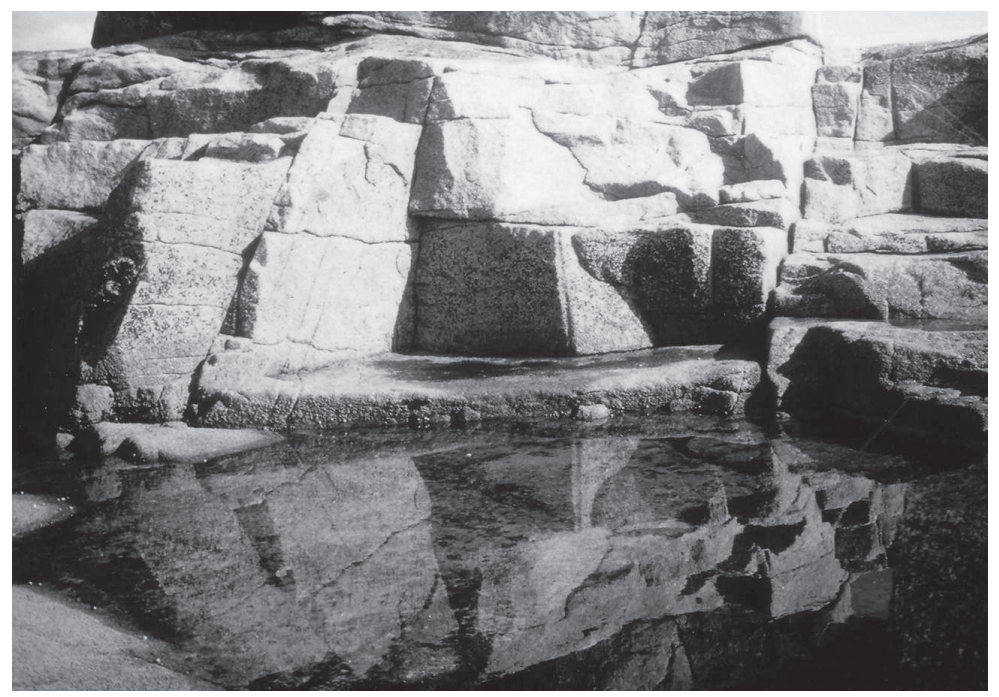




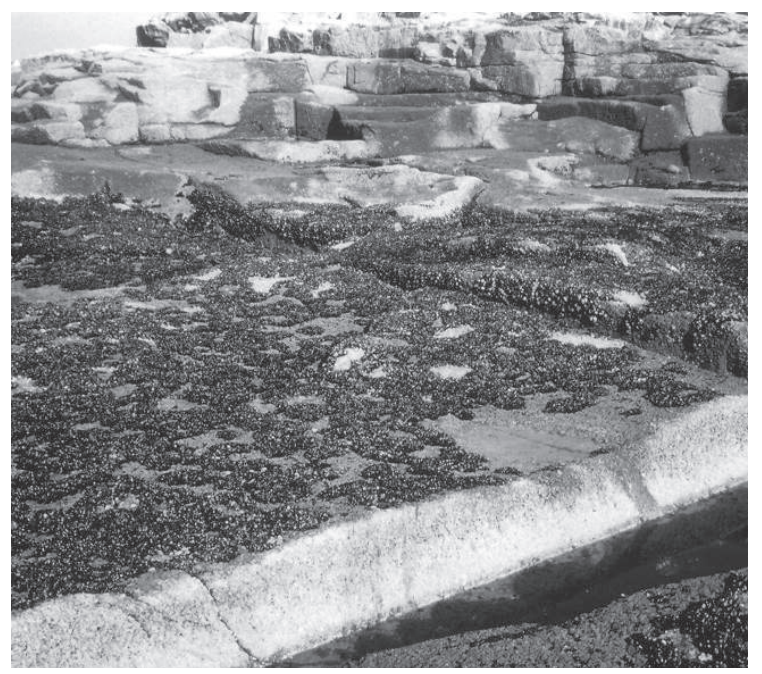

Photo 3-La nature reprend ses droits. Tapis de moules dans une ancienne carrière littorale près de Fort-Bloqué (L. Chauris)

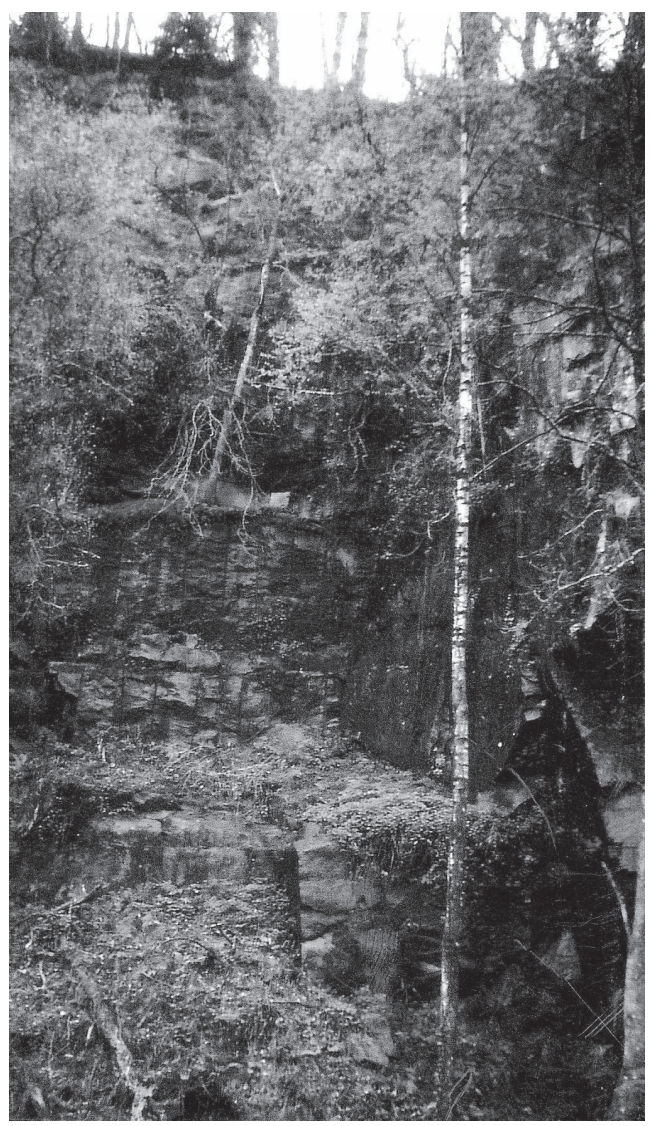

Photo 4 - La carrière de Polvern sur la rive gauche du Blavet, au nord d'Hennebont, disparaît sous la végétation arbustive, laissant à peine percevoir les puissantes masses granitiques du front de taille (L. Chauris) 
Photo 5 - Carrière ouverte sur la rive droite du Blavet près de Kerlomhouarn. La végétation masque peu à peu le front de taille subvertical (L. Chauris)

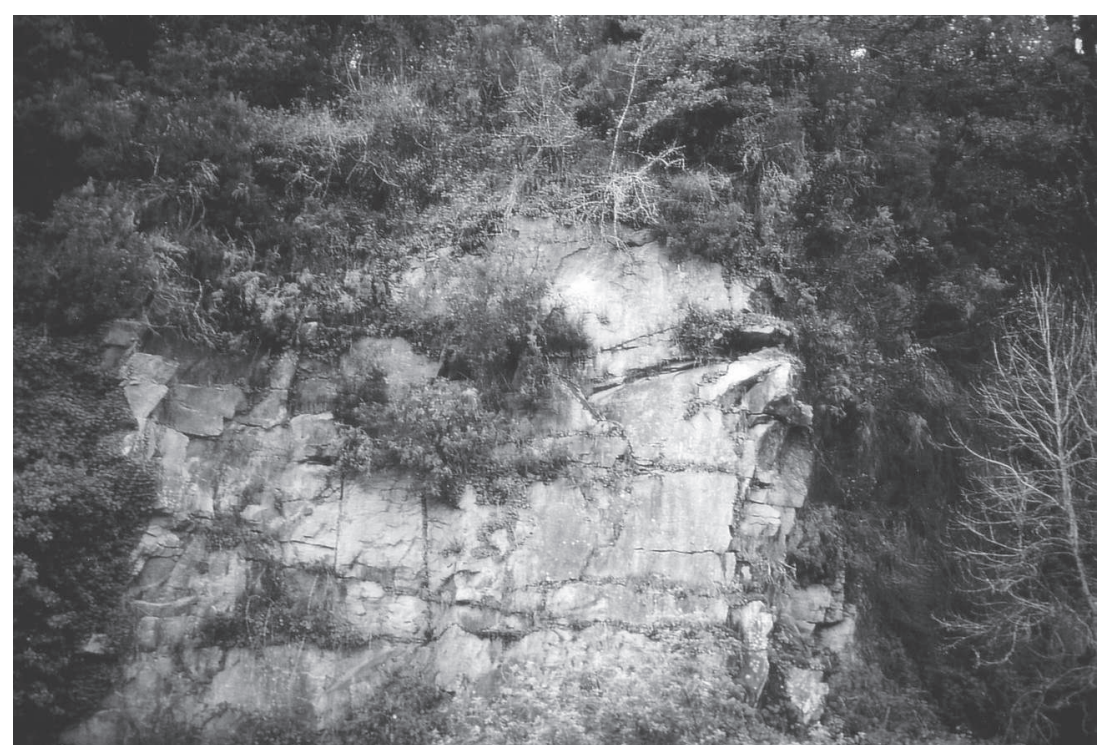




\section{RÉSUMÉ}

La croissance rapide de Lorient à partir du $\mathrm{XVIII}^{\mathrm{e}}$ siècle sous l'impulsion de la Compagnie des Indes, puis de la Marine, induisant la mise en œuvre d'infrastructures urbaines, portuaires et défensives, a entraîné l'exploitation de nombreuses carrières aux environs de la ville. En 1741, un ingénieur du Roi choisit 18 sites en fonction de leur position géographique et de la qualité des matériaux; ce nombre élevé s'interprète par une faible dimension individuelle et par les énormes besoins d'une ville en gestation. Dans la première partie du $\mathrm{XIX}^{\mathrm{e}}$ siècle, est entrepris sans grand succès, un inventaire systématique des carrières... Des prospections sur le terrain ont permis de retrouver de très nombreuses perrières depuis longtemps abandonnées. Les chantiers étaient ouverts en bordure de l'Océan (entre autres sur les rivages de Ploemeur) et sur les versants des rias navigables (le Scorff, le Blavet et la rivière d'Étel). Ces situations offraient deux atouts majeurs : facilité de l'extraction sans coûteux travaux de " découverte " et possibilité d'acheminement par voie d'eau aux époques où les charrois s'avéraient pénibles et dispendieux. Toutefois, les environs de Lorient n'ont pu fournir toutes les pierres nécessaires, d'où appel à d'autres matériaux bretons, en particulier au granite de Trégunc dans le Finistère; déjà au XVIII ${ }^{\mathrm{e}}$ siècle, aux " pierres dures " bretonnes (granites) venaient s'adjoindre les " pierres tendres " (calcaires) du Val de Loire et de la Charente. Dans le cadre de la sauvegarde du patrimoine d'archéologie industrielle est suggérée la " réhabilitation " d'un des sites les plus célèbres (Polvern).

\section{ABSTRACT}

The rapid growth of Lorient from the eighteenth century onwards, under impulse of the "Compagnie des Indes » and later of the French navy, led to town planning, harbour installations and defensive works. As a result, it was also necessary to develop numerous quarries in the vicinity of the town. In 1741, a royal engineer selected 18 quarries according to their geographical location and the quality of the stone. The high number of sites is explained by their small size and by the considerable needs of a burgeoning town. In the first part of nineteenth century, a systematic inventory of the quarries was undertaken, but without much result. Fieldwork has allowed us to rediscover a large number of quarries that had been deserted or even filled in. These sites were opened along the sea (Ploemeur) and on the sides of navigable rias (Scorff, Blavet, Etel). These positions offered two great advantages: they made quarrying easier and the stones could be transported by boat. Nevertheless, local quarries could not provide all the required stones and it was necessary to bring in other building materials, in particular granite from Trégunc in Finistère. From the very start, the hard Breton stones (granite) were complemented by soft stones (limestone) from the Loire valley and Charente. Today, it is suggested that the famous Polvern quarry should be "rehabilitated" in order to preserve some of the industrial archaeological heritage. 